INPLASY

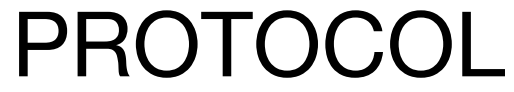

To cite: Xue et al. Chronic ankle instability is associated with proprioception deficits: A systematic review with metaanalysis. Inplasy protocol 202040125. doi:

10.37766/inplasy2020.4.0125

Received: 21 April 2020

Published: 21 April 2020

Corresponding author:

Yinghui Hua

hua_cosm@aliyun.com

Author Affiliation:

Huashan Hospital, Fudan

University

Support: NNS Found of China

Review Stage at time of this submission: Formal screening of search results against eligibility criteria.

Conflicts of interest: None.

\section{Chronic ankle instability is associated with proprioception deficits: A systematic review with meta-analysis}

\author{
Xue, XA1; Ma, TJ2; Li, QR ${ }^{3}$; Song, YJ4; Hua, YH5.
}

Review question / Objective: Whether deficits of proprioception, including kinesthesia and joint position sense (JPS), exist in CAI patients compared with the uninjured contralateral limbs or healthy people.

Condition being studied: Lateral Ankle sprain (LAS) is one of the most common sport-related lower extremity musculoskeletal injury. Over 2 million ankle sprains are treated in emergency departments in the US and UK each year, resulting in about $\$ 2$ billion of healthcare costs. In longterm prognosis, more than $30 \%$ of patients reporting repetitive bouts of ankle giving way and recurring sprains, termed as chronic ankle instability. For the patients suffering from persistent symptoms, surgery is usually suggested and most of them can obtain good outcomes, while some still end up poorly and fail to return to sport. One possible reason can be the sensorimotor deficits, including impaired proprioception, caused by the disfunction of mechanoreceptors within the injured ankle.

INPLASY registration number: This protocol was registered with the International Platform of Registered Systematic Review and Meta-Analysis Protocols (INPLASY) on 21 April 2020 and was last updated on 21 April 2020 (registration number INPLASY202040125.

\section{INTRODUCTION}

Review question / Objective: Whether deficits of proprioception, including kinesthesia and joint position sense (JPS), exist in CAI patients compared with the uninjured contralateral limbs or healthy people.
Condition being studied: Lateral Ankle sprain (LAS) is one of the most common sport-related lower extremity musculoskeletal injury. Over 2 million ankle sprains are treated in emergency departments in the US and UK each year, resulting in about $\$ 2$ billion of healthcare costs. In long-term prognosis, more than $30 \%$ of patients reporting repetitive bouts of ankle giving way and recurring sprains, 
termed as chronic ankle instability. For the patients suffering from persistent symptoms, surgery is usually suggested and most of them can obtain good outcomes, while some still end up poorly and fail to return to sport. One possible reason can be the sensorimotor deficits, including impaired proprioception, caused by the disfunction of mechanoreceptors within the injured ankle.

Rationale: Individuals with chronic ankle instability (CAI) may have the impairments of proprioception owing in part to injured mechanoreceptors in ligaments, while the results of current studies are still in conflict.

\section{METHODS}

Search strategy: Search strategies for PubMed () : Ankle-related terms: (ankle* OR talo* OR tibiofib* OR tibio-fib* OR "lateral ligament" OR "lateral ligaments") Injury-related terms: ("ankle injuries"[MESH] OR unstable OR instabilit* OR strain* OR sprain* OR rupture* OR tear*) Proprioception-related terms: (propriocep* OR sensor* OR sensa* OR sense $^{\star}$ OR feedback ${ }^{\star}$ OR movement ${ }^{\star}$ OR motion* OR kinetic* OR kinematic* OR kinesthe* OR position*) Search 'Anklerelated terms', 'Injury-related' terms and 'Proprioception-related terms' linked with "AND".

Participant or population: Chronic ankle instability patients.

Intervention: None.

Comparator: Healthy control or Contralateral healthy limb.

Study designs to be included: Observational Studies.

Eligibility criteria: The inclusion criteria were as follows: peer-reviewed human studies in English that investigated deficits of kinesthesia and joint position sense and in individuals with a history of ankle sprain and one of the symptoms, including "giving way", recurrent sprains and self-reported instability, compared with either the uninjured contralateral side or healthy controls. For the studies with interventions (e.g. taping, treatment or fatigue), the data without any intervention (e.g. baseline or non-intervention control) should be reported. If the studies mixed the joint movement directions of tests, or included bilateral injured people in between limbs comparison, or enrolled participants with other lower limb pathologies or neurological disorders, they would be excluded.

Information sources: Seven electronic database, including Embase, Web of Science, SPORTDiscus, PubMed, Scopus, CINAHL and Cochrane Library. The reference lists of each included paper were also checked manually. The authors were contacted if the full text was not available.

Main outcome(s): There are three main testing techniques for assessing proprioception: threshold to detection of passive motion (TTDPM), joint position reproduction (JPR) and active movement extent discrimination assessment (AMEDA). The following outcomes were evaluated: (1) TTDPM: Participants were asked to press a stop button as soon as they perceive the movement and direction and then report the perceived direction of movement of their ankle. (2) JPR: Participants were presented with a predetermined target joint position passively or actively for a few seconds, then the joint is returned to the initial position. Participants are then required to reproduce that target joint position. (3) AMEDA: Participants were asked to experience some movement displacement distances with specific position numbers before the test. Then they were presented the experienced positions in a random order and asked to judge the position number. There are three main testing techniques with specific outcomes. (1) TTDPM: The difference between the angle at which participants perceived the movement and the angle of starting point. (2) JPR: The difference between the angle indicated by the patient and the target 
angle. (3) AMEDA: The area under the curve of operating characteristic (ROC) analysis (AUC).

\section{Additional outcome(s): None.}

Data management: Studies were reviewed independently by two authors (XX, TM). If disagreements couldn't be resolved through discussion, the third reviewer (YH) would be consulted. Following information from included articles: demographic data, sample size, selection criteria, methodology (e.g. comparison type, devices, test posture, joint movement direction, target angle, angular velocity), test results (means and standard deviation) and test reliability. The authors were contacted if numerical data were confusing or not reported.

Quality assessment / Risk of bias analysis: All the authors discussed the standard of each item in detail before formal rating, and two authors (XX, TM) rated the included studies independently. The inter-rater agreement of the initial rating was calculated, and the disagreements was also consulted by the third reviewer (YH). To assess the quality of studies, the epidemiological appraisal instrument (EAI) was applied. The EAI tool included 33 items for observational studies, and each item was scored as 'Yes' ( 1 point), 'Partial' ( 0.5 points), 'No' ( 0 points), and 'unable to determine' ( 0 points). An average score would be calculated as the overall quality. To evaluate the risk of bias, a standardized tool recommended by the NonRandomized Studies Group of the Cochrane Collaboration was applied, and the following items were judged: performance bias, detection bias, attrition bias, selection bias and control of confounding for details of tests and analysis. Publication bias would be quantitatively assessed by Egger's regression test.

Strategy of data synthesis: A meta-analysis of the random-effects model would was performed by Stata V.14 (Stata Corp LP, College Station, TX, USA) for the studies similar in comparison type (e.g. between groups) and movement direction (e.g. inversion). All extracted and pooled data would be presented as standardized mean difference (SMD) between controls and the injured ankle with $95 \%$ confidence intervals (CI). Higher SMD represented larger force sense deficits in CAl, with $0.2 \sim 0.5$ as weak, $0.5 \sim 0.8$ as moderate, $>0.8$ as large-sized effect. To evaluate heterogeneity, $Q$ and 12 statistics were calculated, with $p 0.8$ as almost perfect strength of agreement.

Subgroup analysis: Active and passive test.

Sensibility analysis: Sensitivity analysis would be conducted through removing single study at a time and then evaluating the pooled results again.

Language: English.

Country(ies) involved: China.

Keywords: Chronic ankle instability, Proprioception.

Contributions of each author:

Author 1 - Study design; literature search and selection; data collection; quality rating; statistical analysis; writing of the manuscript.

Author 2 - Study design; literature search and selection; data collection; quality rating; reviewing of the manuscript.

Author 3 - Study design; reviewing the manuscript.

Author 4 - Study design; reviewing the manuscript.

Author 5 - Study design; supervision of literature search, data collection and quality rating; reviewing the manuscript. 